\title{
Diversity of endophytic fungi and screening of fungal paclitaxel producer from Anglojap yew, Taxus $x$ media
}

Zhi-Qiang Xiong ${ }^{1}$, Ying-Ying Yang ${ }^{1,2}, \mathrm{Na}^{2}$ Zhao $^{1}$ and Yong Wang ${ }^{1 *}$

\begin{abstract}
Background: Endophytic fungi represent underexplored resource of novel lead compounds and have a capacity to produce diverse class of plant secondary metabolites. Here we investigated endophytic fungi diversity and screening of paclitaxel-producing fungi from Taxus $x$ media.

Results: Eighty-one endophytic fungi isolated from T. media were grouped into 8 genera based on the morphological and molecular identification. Guignardia and Colletotrichum were the dominant genera, whereas the remaining genera were infrequent groups. The genera Glomerella and Gibberella were first reported in Taxus. Three representative species of the distinct genera gave positive hits by molecular marker screening and were capable of producing taxol which were validated by HPLC-MS. Among these 3 taxol-producing fungi, the highest yield of taxol was $720 \mathrm{ng} / \mathrm{l}$ by Guignardia mangiferae HAA11 compared with those of Fusarium proliferatum HBA29 (240 ng/l) and Colletotrichum gloeosporioides TA67 (120 ng/l). This is the first report of taxol producer from Guignardia. Moreover, the lower similarities of ts and bapt between microbial and plant origin suggested that fungal taxol biosynthetic cluster might be repeatedly invented during evolution, nor horizontal gene transfer from Taxus species.
\end{abstract}

Conclusions: Taxol-producing endophytic fungi could be a fascinating reservoir to generate taxol-related drug lead and to elucidate the remained 5 unknown genes or the potential regulation mechanism in the taxol biosynthesis pathway.

Keywords: Taxus x media, Paclitaxel, Endophytic fungi, Guignardia mangiferae, Taxol gene cluster

\section{Background}

Plant-associated microorganisms, especially endophytic fungi, are largely underexplored in the discovery of natural products [1]. The prolific endophytes also have a capacity to produce diverse class of plant associated secondary metabolites with a wide variety of biological activities such as antimicrobial agent hypericin [2], acetylcholinesterase inhibitor huperzine A [3], and antitumor agents taxol [4]. Bioprospecting endophytes thus offers tremendous promise to discover natural products with therapeutic value [1], which have attracted increasing attention among microbiologists, ecologists, agronomists, and chemists.

\footnotetext{
*Correspondence: yongwang@sibs.ac.cn

${ }^{1}$ Key Laboratory of Synthetic Biology, Institute of Plant Physiology and Ecology, Shanghai Institutes for Biological Sciences, Chinese Academy of Sciences, Shanghai 200032, China

Full list of author information is available at the end of the article
}

Among plant-derived natural products, taxol (a blockbuster anticancer drug) is widely used for clinical application against different types of cancer [5]. It was originally obtained from extraction of the bark of Taxus species. However, mass production of taxol remains a vexing problem due to low taxol content in the Taxus species. 13,500 kg of T. brevifolia (Pacific yew, the most productive species) bark only yields about $1 \mathrm{~kg}$ of taxol [6], whereas at least $2 \mathrm{~g}$ of taxol is required for a full regimen of antitumor treatment in a patient [4]. With the increasing demand for taxol and the shortage of plant resource, there is an urgent need to find other alternative production methods.

Several alternative strategies have been developed for taxol production during the past two decades. Total chemical synthesis is available [7], but the large number of reaction steps and low yield limit its practicality. Semisynthesis from taxol precursors baccatin III or 10- 
deacetylbaccatin III solves the supply problem of taxol which appears so formidable, but still relies on plant precursor compounds with difficulty in the purification process [8]. Plant tissue culture as an environmentally sustainable method is successfully developed for largescale taxol production, but long incubation time and low yield render it an economic impossibility [9]. Notwithstanding the remarkable progress in the different production alternatives, these methods are not enabled to meet the increasing taxol demand with an economic supply [10]. Consequently, more production options are still required to lower the price of taxol and increase its availability.

Taxomyces andreanae is the first report of a microbial taxol producer from Pacific yew [4], implying that microorganisms as a potential source would be one of the most desirable means for taxol supply. Potential advantages of microbial taxol production include a fast growth at high cell density cultivation, easy genetic manipulation, and the possibility of scale-up on an industrial level [10]. In addition, microbial production helps to protect natural plant Taxus resources [11]. Current research in this field is focused on screening taxol-producing endophytic microbes [4], improving taxol yield by genome shuffling [12], genetic engineering [13], and process optimization [14], and heterologous expression of taxol precursor in microorganisms [15].

Isolation of endophytic microorganisms is a comparatively simple process, but taxol detection of all isolates is laborious [16]. Compared to this traditional screening method, the molecular marker screening is an efficient alternative method to find taxol-producing microbes [17]. Three probes based on key genes of taxol biosynthetic cluster, $t s$ (encoding taxadiene synthase), dbat (encoding 10-deacetylbaccatin III-10-O-acetyltransferase), and bapt (encoding $\mathrm{C}-13$ phenylpropanoyl side chain-CoA acyltransferase), have been applied in the primary screening of taxol-producing endophytic microorganisms (Figure 1). Currently, more than 30 endophytic microorganisms have been reported to produce taxol with ranging from $10 \mathrm{ng} / \mathrm{l}$ to $800 \mu \mathrm{g} / \mathrm{l}$ through the traditional screening/molecular marker screening [10]; the majority of endophytic microbes belong to fungi such as ascomycetes and imperfect fungi, and a minority are bacteria such as Kitasatospora and Streptomyces. However, to date, there are only a few reports to investigate biodiversity of microorganisms living in Taxus [18]. In this work, we surveyed the endophytic fungi diversity of T. media, and discovered taxol-producing endophytes from the fungal isolates based on molecular markers derived from key biosynthetic enzymes of taxol. To our knowledge, Guignardia is the first report to produce taxol.

\section{Results and discussion}

\section{Endophytic fungal diversity of $T$. media}

To assess the presence of fungal endophytes in T. media, 81 fungal isolates were recovered and assigned to 29 morphotypes using dereplication based on the morphological characteristics and unique phenotypic characters (Figure 2). The identified fungi belonged to the phylum Ascomycota. To confirm the reliability of morphological identification, all 29 morphotypes (strains HAA3,

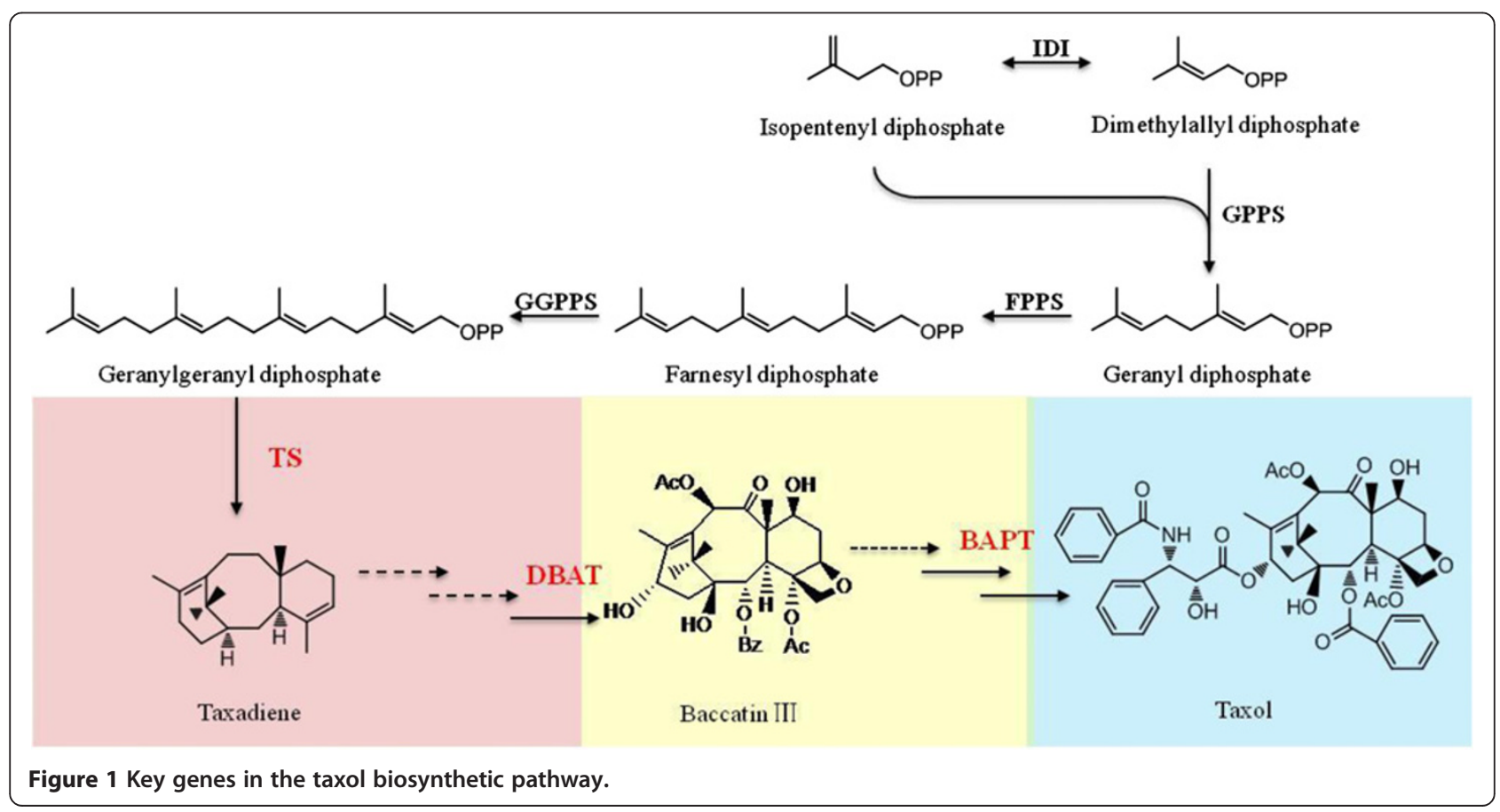



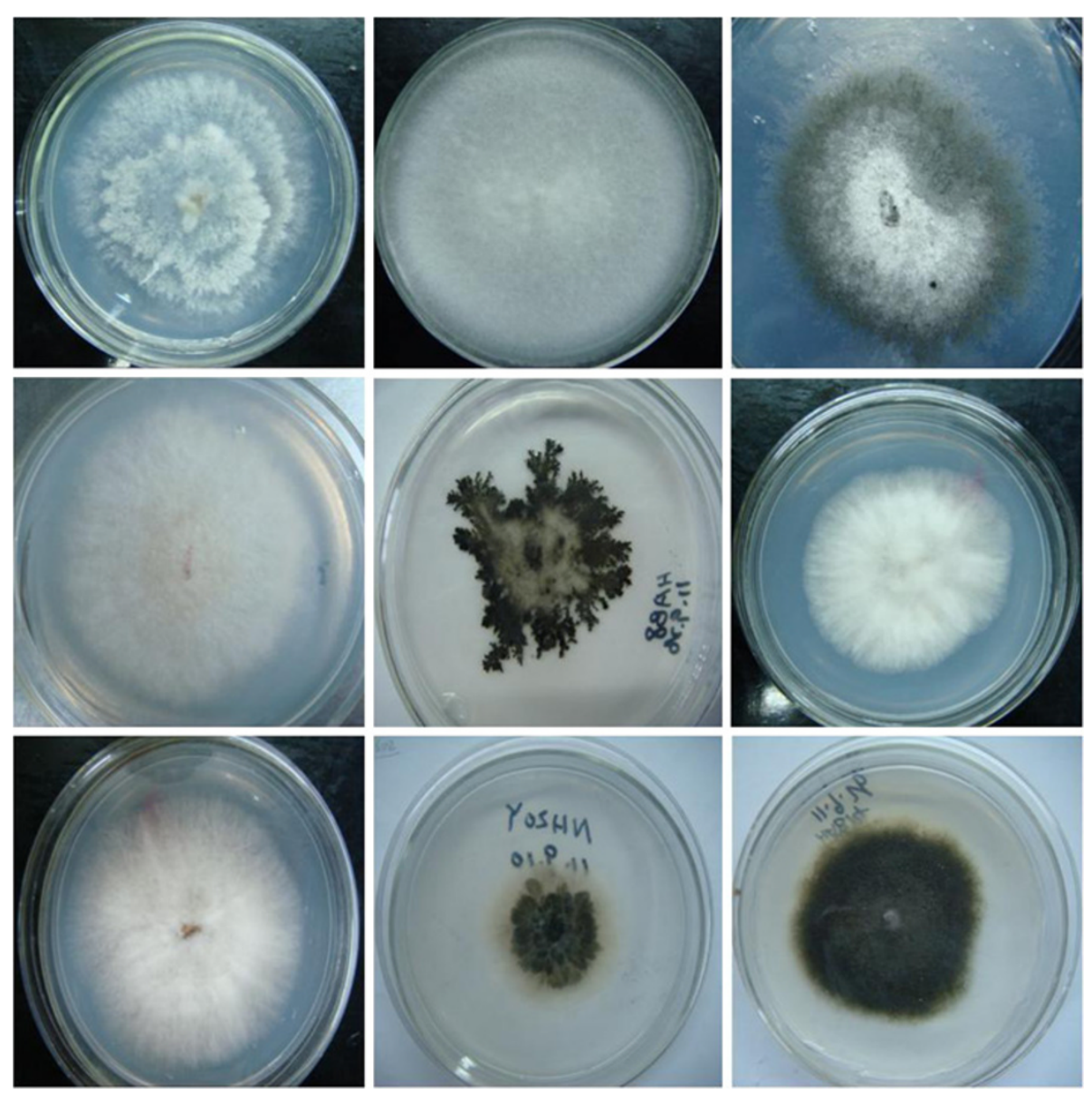

Figure 2 Morphological characteristics of fungal endophytes in T. media.

HAA4, HAA5, HAA7, HAA8, HAA11, HAA12, HAA22, HAA24, HBA6, HBA12, HBA18, HBA26, HBA29, HBA30, HBA31, TA47, TA67, TA235, TA237, TA240, TA242, TA244, TA246, TA247, TA250, TA252, TA255, and TA278) were subjected to molecular identification based on ITS rDNA sequence analysis (Figure 3). The 29 morphospecies were grouped into 8 genera (Alternaria, Colletotrichum, Glomerella, Gibberella, Guignardia, Nigrospora, Phomopsis, and Phoma). Analysis of distribution frequencies of the 29 morphotypes revealed that the fungal communities in the host contained two frequent genera and many infrequent groups (Figure 4). Glomerella and Colletotrichum were the dominant genera, accounting for $13.8 \%$ and $58.6 \%$ of colonization frequencies (Table 1). Among the rare genera, Alternaria and Guignardia represented $\sim 6.9 \%$ of isolation frequencies, whereas others showed $\sim 3.4 \%$ of colonization frequencies (Table 1). Our result confirmed that a few species are frequent colonizers, and yet the majority are rare inhabitants in woody plants [18].

Although Glomerella and Colletotrichum are frequent colonizers in T. media (temperate regions) in this study, they are not cosmopolitan species within other Taxus plants [18,19], such as the frequent genera Diaporthe, Phomopsis, Acremonium, and Pezicula in T. chinensis (mountain region of Qinba, northern-western China), and Myceliasterilia, Alternaria, and Fusarium in $T$. baccata and $T$. brevifolia (central-northern Italy), indicating that the dominant genera are distinct in different yews and different geographic region [20]. The genera Glomerella and Gibberella were first reported endophytes in Taxus, but they have been isolated from other host plants [21,22].

In total, 11 distinctive genotypes were detected at a 99\% sequence similarity threshold (Figure 3), which did not correspond well with morphological differences between these fungal cultures. Strains HAA12, HBA29, TA47, TA244, TA246, and TA278 were located with a high bootstrap support (99-100\%) in their own cluster, while strains HAA11, HAA22, HBA18, TA67, TA235, TA237, TA240, TA242, TA250, and TA255 formed their own cluster with a bootstrap value from 70 to $99 \%$. Strains HAA3, HAA4, HAA5, HAA7, HAA8, HAA24, HBA6, HBA12, HBA26, HBA30, and HBA31 were clustered to Colletotrichum 


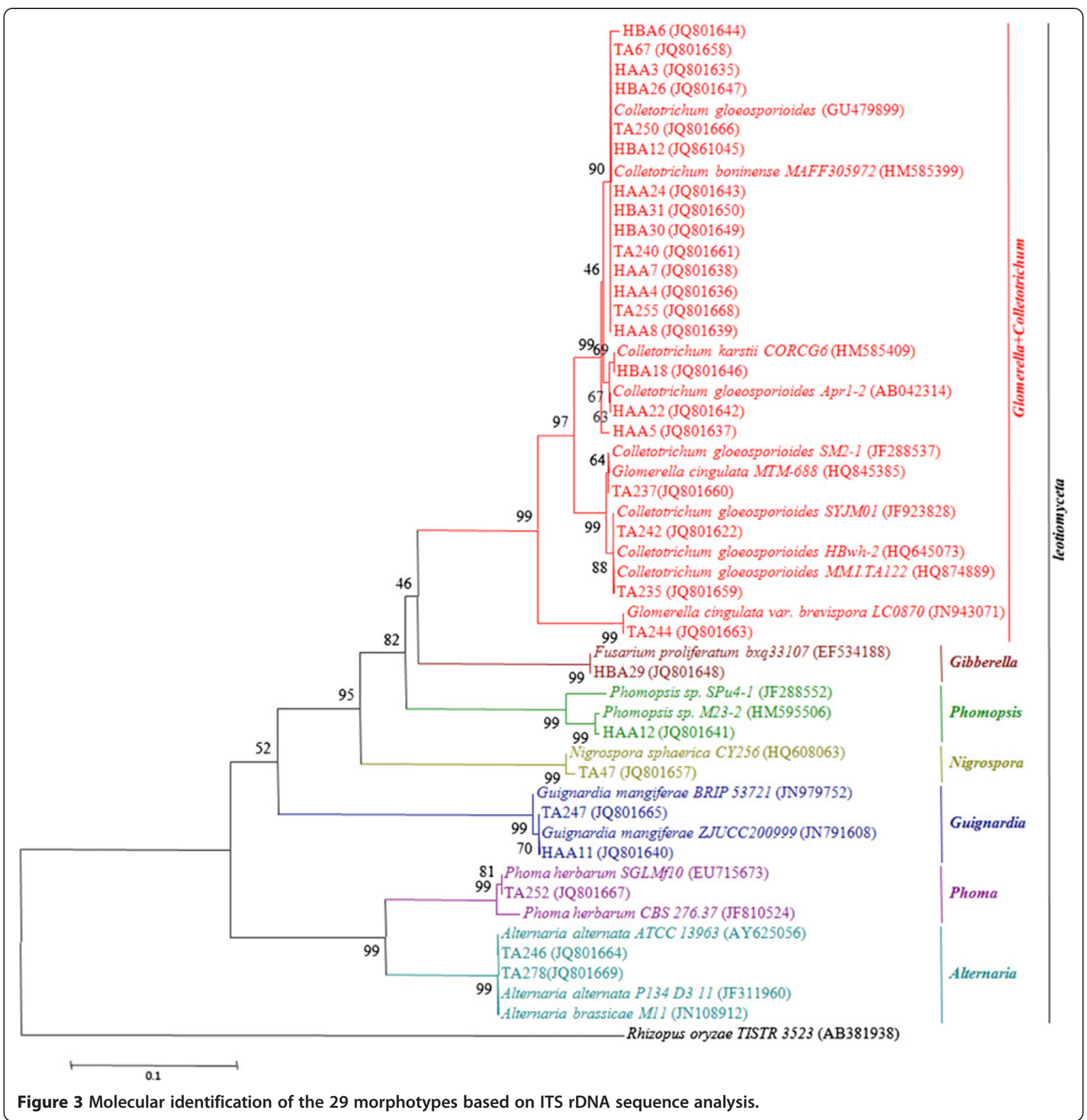

boninense with a bootstrap value of $90 \%$, but sequence identities with the available references in NCBI were very high $(100 \%)$. In addition, strain HAA5 was not similar to any references with a bootstrap value of $63 \%$, but shared sequence similarities of $99 \%$ with $C$. boninens. It might represent novel species or even new genera.

\section{Primary screening of taxol-producing fungi based on molecular marker}

Molecular marker based screening is a rapid and efficient alternative to find taxol-producing endophytic microbes in contrast to the traditional screening method $[11,17]$. This method is not dependent on the production of paclitaxel and can indicate the presence of some required genes for taxol biosynthesis in the microbial genome. In yew trees, taxol biosynthesis involves 19 enzymatic steps from the universal diterpenoid precursor geranylgeranyl diphosphate (GGPP) by the plastidial methyl erythritol phosphate pathway [23]. We thus chose $t s$ (involved in formation of the taxane skeleton), dbat (involved in baccatin III formation), and bapt (involved in phenylpropanoyl side chain formation at C13), three key 


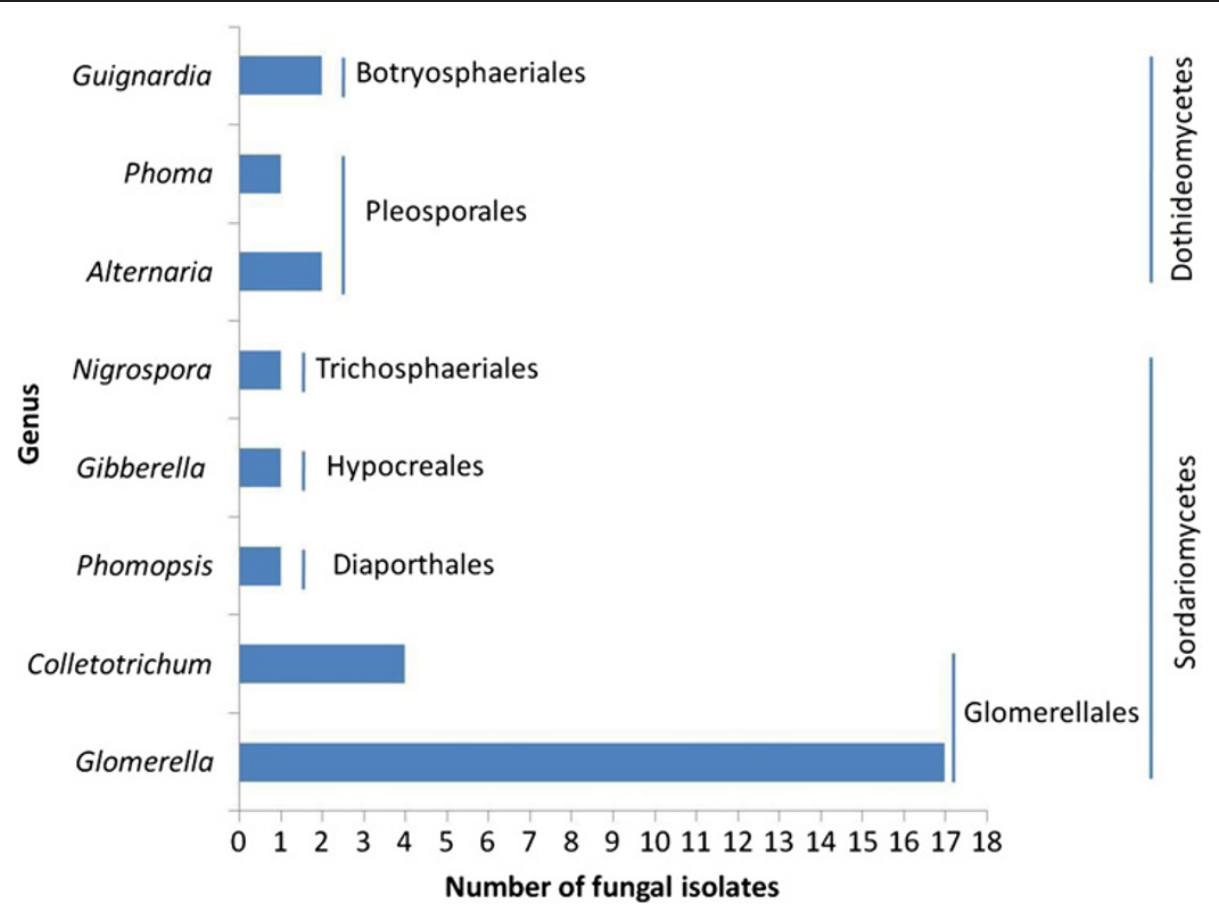

Figure 4 The frequency of ITS-based genotypes determined from the 29 morphotypes.

genes in taxol biosynthesis, as a primary screening to identify taxol-producing fungi.

All 11 fungal isolates with distinctive genotype separated from $T$. media were consecutively screened for the presence of $t s, d b a t$, and bapt genes. Three fungi (strains HAA11, HBA29, and TA67) had positive hits of $t s$ and $d b a t$. The $t s$ and dbat genes are essential for taxol biosynthesis but not diagnostic because taxol precursor baccatin III producers also have $t s$ and dbat. Thus, the 3 fungi were screened for the presence of bapt. Interestingly, all these 3 fungi had approximately 530 bp fragments of bapt gene (Figure 5), suggesting that all of them may produce taxol. Currently, only $t s$, $d b a t$, and bapt genes have been used as molecular probes for the primary screening of taxol producing microorganisms $[16,17]$, thus designing suitable degenerate primers for amplification of more target genes, e.g., the final acylation step in taxol biosynthesis, taxoid C13-side-chain $\mathrm{N}$ benzoyltransferase (DBTNBT), may be a better option for screening.

\section{Identification of fungal taxol}

We screened the extracts of the 3 representative species Guignardia mangiferae HAA11, Fusarium proliferatum HBA29, and Colletotrichum gloeosporioides TA67 with positive results in the primary screening to detect fungal taxol by high performance liquid chromatography-mass spectrometry (LC-MS). The HPLC peak positions and peak shapes of the 3 representative species from the different genera were identical to that of standard taxol (retention time $=21.02 \pm 0.03 \mathrm{~min}$ ), indicating the 3 distinct fungi may produce taxol. Further convincing evidence for the identity of the fungal taxol was obtained by high resolution MS (Figure 6). Characteristically, the authentic taxol yielded an $[\mathrm{M}-\mathrm{H}]^{-}$peak at $\mathrm{m} / z 852.32$ and an $[\mathrm{M}+\mathrm{COOH}]^{-}$peak at $m / z$ 898.32. By comparison, the fungal taxol also yielded a peak at $m / z 852.32 \pm 0.03$ and a characteristic fragment peak at $m / z 898.32 \pm 0.02$ (Table 2). The peaks of fungal taxol exhibited $\mathrm{m} / z$ ratios corresponding to the molecular ions of standard taxol, demonstrating that the 3 fungal endophytes can generate taxol in vitro. Among these 3 taxol-producing fungi, strain HAA11 had the highest taxol yield $(720 \mathrm{ng} / \mathrm{l})$ in the PDB medium in comparison with those of strains HBA29 (240 ng/l) and TA67 (120 ng/l).

Colletotrichum gloeosporioides has been proven to be capable of producing taxol $(163.4 \mu \mathrm{g} / \mathrm{l})$ [24]. Guignardia mangiferae and Fusarium proliferatum have not been obtained from other yews and some reported taxolproducing fungi from other Taxus plants have not been isolated from T. media in this work, suggesting that yews in different geographic regions can harbor novel and highly diverse taxol producing fungi and certain taxol-generating fungi may be host-specific. Thus, to isolate taxol-producing fungal species, more consideration should be given to different hosts under different conditions. In addition, Guignardia mangiferae HAA11 and Fusarium proliferatum HBA29 were recovered as 
Table 1 Putative taxonomic affinities and frequency of the $\mathbf{2 9}$ morphotypes

\begin{tabular}{|c|c|c|c|c|c|}
\hline Fungal isolate & Accession number & Closest relatives in $\mathrm{NCBI}$ & ITS identity (\%) & Frequency & Genus \\
\hline HAA3 & JQ801635 & Colletotrichum boninense MAFF305972 (HM585399) & $100 \%$ & $34.5 \%$ & Colletotrichum \\
\hline HAA4 & JQ801636 & Colletotrichum boninense MAFF305972 (HM585399) & $100 \%$ & & \\
\hline HAA7 & JQ801638 & Colletotrichum boninense MAFF305972 (HM585399) & $100 \%$ & & \\
\hline HAA8 & JQ801639 & Colletotrichum boninense MAFF305972 (HM585399) & $100 \%$ & & \\
\hline HAA24 & JQ801643 & Colletotrichum boninense MAFF305972 (HM585399) & $100 \%$ & & \\
\hline $\mathrm{HBA} 12$ & JQ801645 & Colletotrichum boninense MAFF305972 (HM585399) & $100 \%$ & & \\
\hline HBA26 & JQ801647 & Colletotrichum boninense MAFF305972 (HM585399) & $100 \%$ & & \\
\hline HBA30 & JQ801649 & Colletotrichum boninense MAFF305972 (HM585399) & $100 \%$ & & \\
\hline HBA31 & JQ801650 & Colletotrichum boninense MAFF305972 (HM585399) & $100 \%$ & & \\
\hline HBA6 & JQ801644 & Colletotrichum boninense MAFF305972 (HM585399) & $99 \%$ & & \\
\hline HAA5 & JQ801637 & Colletotrichum petchii CBS:378.94 (JQ005223) & $99 \%$ & $3.4 \%$ & \\
\hline HBA18 & JQ801646 & Colletorichum karstii CORCG6 (HM585409) & $100 \%$ & $3.4 \%$ & \\
\hline TA67 & JQ801658 & Colletotrichum gloeosporioides (GU479899) & $100 \%$ & $17.2 \%$ & \\
\hline TA240 & JQ801661 & Colletotrichum gloeosporioides (GU479899) & $99 \%$ & & \\
\hline TA250 & JQ801666 & Colletotrichum gloeosporioides (GU479899) & $100 \%$ & & \\
\hline TA255 & JQ801668 & Colletotrichum gloeosporioides (GU479899) & $99 \%$ & & \\
\hline TA242 & JQ801662 & Colletotrichum gloeosporioides MM.I.TA122 (HQ874889) & $100 \%$ & & \\
\hline HAA11 & JQ801640 & Guignardia mangiferae ZJUCC200999 (JN791608) & $100 \%$ & $6.9 \%$ & Guignardia \\
\hline TA247 & JQ801665 & Guignardia mangiferae ZJUCC200999 (JN791608) & $100 \%$ & & \\
\hline HAA12 & JQ801641 & Phomopsis sp. M23-2 (HM595506) & $99 \%$ & $3.4 \%$ & Phomopsis \\
\hline HAA22 & JQ801642 & Glomerella sp. HS-EF2 (GQ334409) & $100 \%$ & $3.4 \%$ & Glomerella \\
\hline TA237 & JQ801660 & Glomerella cingulata MTM-688 (HQ845385) & $100 \%$ & $10.3 \%$ & \\
\hline TA235 & JQ801659 & Glomerella cingulata MAFF 305913 (AB042315) & $99 \%$ & & \\
\hline TA244 & JQ801663 & Glomerella cingulata var. brevispora LC0870 (JN943071) & $100 \%$ & & \\
\hline HBA29 & JQ801648 & Fusarium proliferatum bxq33107 (EF534188) & $100 \%$ & $3.4 \%$ & Gibberella \\
\hline TA47 & JQ801657 & Nigrospora sphaerica CY256 (HQ608063) & $99 \%$ & $3.4 \%$ & Nigrospora \\
\hline TA246 & JQ801664 & Alternaria brassicae M11 (JN108912) & $100 \%$ & $3.4 \%$ & Alternaria \\
\hline TA278 & JQ801669 & Alternaria alternata P143_D3_11 (JF311960) & $100 \%$ & $3.4 \%$ & \\
\hline TA252 & JQ801667 & Phoma herbarum SGLMf10 (EU715673) & $99 \%$ & $3.4 \%$ & Phoma \\
\hline
\end{tabular}

infrequent genera, indicating that infrequent genera from Taxus might be a huge source of taxol-producing fungi [18].

Although taxol concentration of Guignardia mangiferae HAA11, Fusarium proliferatum HBA29, and Colletotrichum gloeosporioides TA67 is relatively lower than that of Taxus species, the high growth rate and short generation time make them worthwhile to continue investigation. Thus, to meet the commercial need for taxol, further work will focus on improving taxol yield in fungi by combination of various biotechnological approaches such as strain improvement, genetic manipulation, and fermentation engineering. In addition, the lack of a complete taxol biosynthetic cluster (5 unknown enzymatic steps) is at present a bottleneck for basic and applied research, genome sequencing and analysis of taxol-producing microorganisms (the relatively small genomes) thus could significantly expand the number of known taxol biosynthetic genes to elucidate the whole pathway and provide the basis for heterologous production. From an evolutionary adaptation point of view, endophytic microorganism harboring particular metabolic functions could be achieved extraordinary success in occupying a niche within plant tissues or even contribute to host defense against various invading pathogens [18], hence, analysis of genes involved in taxol synthesis from the diverse fungi will significantly enhance our understanding of the co-evolutionary mechanism of the endophyte host [11].

\section{ts and bapt genes between taxol-producing fungi and Taxus}

The amplified DNA fragments of ts (from strain HBA29) and bapt (from strains HAA11 and TA67) were sequenced and analyzed using Blastn in the NCBI 


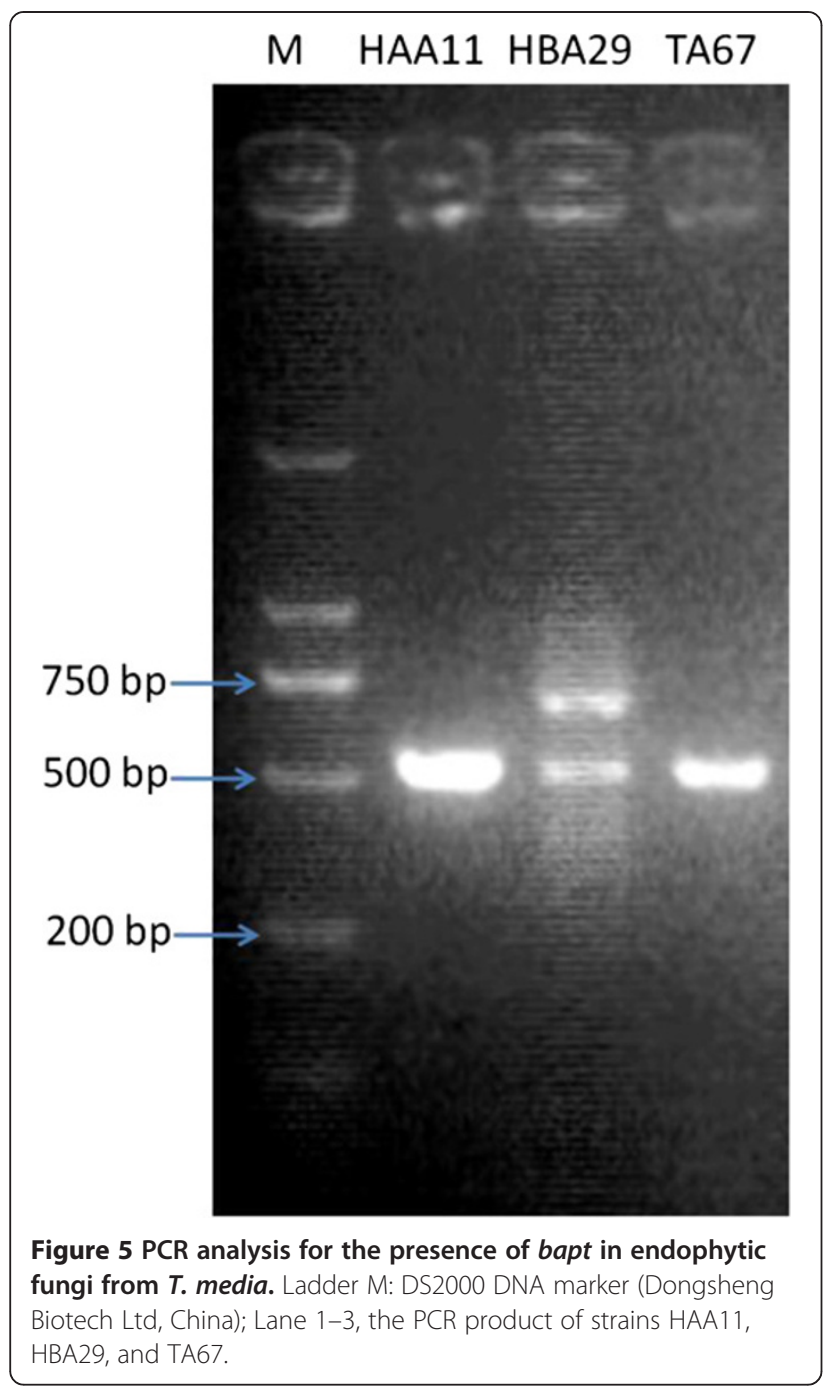

database. The ts segment from strain HBA29 shares $40.6 \%$ identity with cDNA of $t$ f from T. media [GenBank accession no. AY461450]. The bapt segments from strains HAA11 and TA67 have lower identity $(40.0 \%$ and $44.1 \%$, respectively) with cDNA of bapt from $T$. media [GenBank accession no. AY563630], indicating that it might be a fragment of the new putative fungal bapt gene. Despite our findings are contrary to all previous works of ts and bapt from endophytic fungi which show high homology (> 96\% sequence identity) with theirs plant counterparts [10,16,25-27], the success of our screening for microbial $t s$, dbat and bapt using the designed PCR primer based on the conserved regions of key genes of taxol biosynthetic pathway in yew provides crucial evidence for the molecular blueprint of taxol biosynthesis being an inherent genetic trait of endophytic fungi. Moreover, the detection of taxol production affords definitive proof for the presence of taxol pathway in endophytic fungi. Consequently, low similarity of ts and bapt between plant and microbial origin seems to give a new insight to the controversial hypothesis of horizontal gene transfer (HGT). The evolutionary trajectory of taxol gene cluster between microbial and plant origin might be coexisting. Although HGT in fungi are largely reported [28], the ultimate plausibility of microbial taxol gene cluster by HGT hypothesis should be revisited and further investigated because approximately 20 genes involved in the taxol biosynthesis make HGT rather unlikely. Additionally, taxol-producing endophytic fungi have been isolated from plants which themselves are not capable of producing taxol [29-34], suggesting that taxol biosynthesis in fungi may not be acquired from HGT. In nature, gibberellin biosynthetic pathways in fungi and higher plants have evolved independently and not by HGT $[35,36]$. We thus assumed that taxol biosynthetic cluster might be repeatedly invented during evolution. Moreover, it raises an intriguing question: whether the genes responsible for fungal taxol biosynthesis are indeed grouped in a contiguous cluster?

\section{Conclusions}

Eighty-one endophytic fungi isolated from T. media were grouped into 8 genera based on the morphological and molecular identification. Guignardia and Colletotrichum were the dominant genera, whereas the remaining genera were infrequent groups. Three representative species of the distinct genera can produce taxol. This is the first report of taxol prodcer from Guignardia. The highest taxol yield was $720 \mathrm{ng} / \mathrm{l}$ by Guignardia mangiferae HAA-11. Moreover, the lower similarities of ts and bapt between microbial and plant origin indicated that fungal taxol biosynthetic cluster might be repeatedly invented during evolution, nor HGT from Taxus species.

\section{Methods}

\section{Isolation of endophytic fungi from T. media}

Plant samples including the bark pieces and leaves were collected from T. media (Shanghai, China). The samples were treated with $75 \%$ ethanol (v/v) for $1 \mathrm{~min}$ and $2.5 \%$ sodium hypochlorite $(\mathrm{v} / \mathrm{v})$ for $2 \mathrm{~min}$, and rinsed two times in sterilized water. In order to test the effectiveness of surface sterilization [21], sterilized samples were imprinted onto potato dextrose agar with $100 \mu \mathrm{g} / \mathrm{l}$ streptomycin (PDAS) in Petri dishes at $28^{\circ} \mathrm{C}$ for 1 week. In addition, $10 \mathrm{ml}$ of the last rinsing water were centrifuged for $10 \mathrm{~min}$ at $5000 \mathrm{rpm}$. The supernatant was removed and added $500 \mu \mathrm{l}$ sterilized water in the centrifugal tube; $100 \mu \mathrm{l}$ of this volume were then plated onto PDAS. The surface sterilization was validated because no mycelial growth occurred. The surfacedisinfected small pieces $\left(4 \mathrm{~mm}^{2}\right)$ of inner bark and leaf segments were excised and placed on the surface of PDAS in Petri dishes, incubated at $28^{\circ} \mathrm{C}$ for $3-7$ days to allow the growth of endophytic fungi, and periodically 


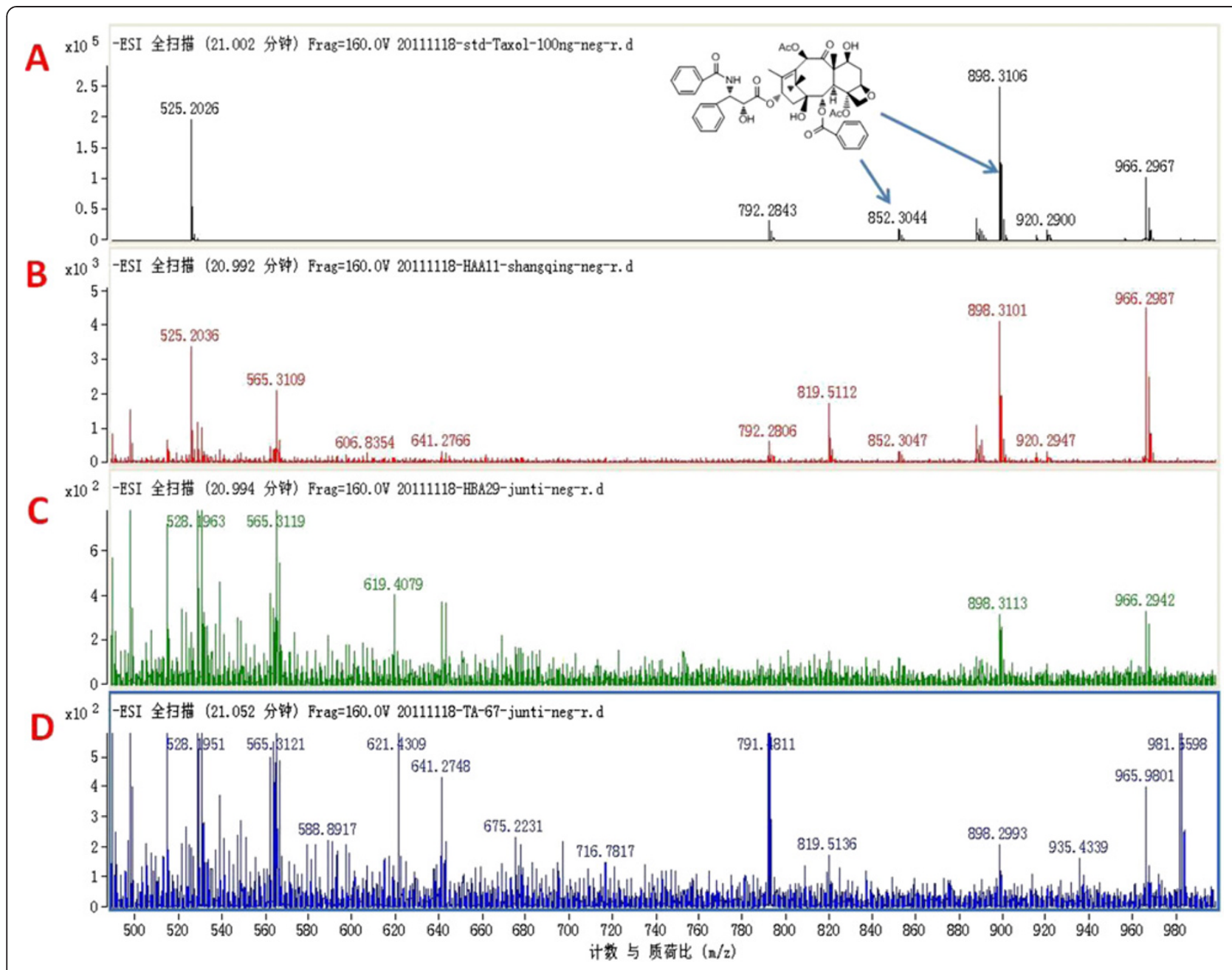

Figure 6 Mass spectrometric analysis of authentic taxol (A) and the fungal isolates sample solution of HAA11 (B), HBA29 (C), and TA67 (D). The arrows indicate the identical peak of mass spectroscopy of taxol.

checked for culture purity. Pure fungal cultures of the endophytic isolates were obtained by the hyphal tip method [37]. All fungal isolates were numbered and stored in $15 \%(\mathrm{v} / \mathrm{v})$ glycerol at $-80^{\circ} \mathrm{C}$ as spores and mycelium.

\section{Identification of endophytic fungi from $T$. media}

Individual hyphal tips of various fungal isolates were subcultured onto fresh PDA medium, and incubated at $28^{\circ} \mathrm{C}$ for at least 2 weeks. All fungal isolates were initially identified to the genus and/or species level based on

Table 2 The mass spectral fragment ions of taxol

\begin{tabular}{cccccccc}
\hline \multicolumn{8}{c}{ Fragment peak } \\
\hline \multicolumn{7}{c}{ Standard } & \multicolumn{2}{c}{ HAA-11 } & \multicolumn{2}{c}{ HBA-29 } & \multicolumn{2}{c}{ TA-67 } \\
\hline$(\mathrm{M}-\mathrm{H})^{-}$ & $(\mathrm{M}$ & $(\mathrm{M}-\mathrm{H})^{-}$ & $(\mathrm{M}$ & $(\mathrm{M}-$ & $(\mathrm{M}$ & $(\mathrm{M}-$ & $(\mathrm{M}$ \\
& $+\mathrm{COOH})^{-}$ & & $+\mathrm{COOH})^{-}$ & $\mathrm{H})^{-}$ & $+\mathrm{COOH})^{-}$ & $\mathrm{H})^{-}$ & $+\mathrm{COOH})^{-}$ \\
852.32 & 898.32 & 852.29 & 898.30 & - & 898.30 & - & 898.31 \\
\hline
\end{tabular}

morphology of fungal colony, characteristics of fungal spore, and molecular phylogenetic analysis. The fungal isolates were inoculated individually into $250 \mathrm{ml}$ Erlenmeyer flasks containing $25 \mathrm{ml}$ potato dextrose broth (PDB) medium. Cultures were incubated at $200 \mathrm{rpm}$ at $28^{\circ} \mathrm{C}$ for 2 days and harvested by centrifugation at $12000 \mathrm{r} / \mathrm{min}$ for $10 \mathrm{~min}$.

Genomic DNA was extracted from 0.5-1 g chilled mycelia in liquid nitrogen using the SDS-CTAB method [38]. The fungal internal transcribed spacer (ITS) fragments (ITS1-5.8S-ITS2 rDNA) were amplified by PCR using the universal primers ITS1 and ITS4 (Table 3). The PCR reaction mixtures $(25 \mu \mathrm{l})$ consisted of $1 \mu \mathrm{l}$ genomic DNA ( 100 ng), $0.5 \mu \mathrm{l}$ forward and reverse primers $(20 \mu \mathrm{M})$, and $12.5 \mu \mathrm{l}$ Premix Taq (TaKaRa Biotechnology Ltd., China), and $10.5 \mu \mathrm{l}$ PCR quality water. The PCR reaction programs were pre-heating at $94^{\circ} \mathrm{C}$ for $3 \mathrm{~min}, 30$ cycles of $94^{\circ} \mathrm{C}$ for $30 \mathrm{~s}, 55^{\circ} \mathrm{C}$ for $30 \mathrm{~s}, 72^{\circ} \mathrm{C}$ for $1 \mathrm{~min}$, and a final extension at $72^{\circ} \mathrm{C}$ for $5 \mathrm{~min}$. The 
Table 3 Oligonucleotide primers used in PCR screening

\begin{tabular}{llll}
\hline Gene (GenBank No.) & Primers & Sequence (5'-3') & Amplicon length \\
\hline ITS1-5.8S-ITS2 rDNA & ITS1 & TCCGTAGGTGAACCTGCGG & $500-600$ bp [39] \\
ts (AY364469) & ITS4 & TCCTCCGCTTATTGATATGC & 631 bp [16] \\
dbat (EF028093) & ts-F & CAAACCCATGTCGAATTGAGAAG & 153 bp [17] \\
bapt (AY082804) & ts-R & CAAGTTGCATACACTCTGGAATCT & 453 bp [17] \\
& dbat-F & GGGAGGGTGCTCTGTTG & GTACCTGAACCACCAGAGG \\
& bapt-F & CCTCTCTCCGCCATTGACAA & TCGCCATCTCTGCCATACTT \\
\hline
\end{tabular}

PCR products were analyzed by agarose gel electrophoresis and purified using a DNA gel exaction kit (Axygen Biotechnology Ltd., China). The purified PCR product was directly sequenced using the same primers by BGI-Shanghai (Shanghai, China).

The ITS sequences of endophytic fungi were compared with the data in National Center for Biotechnology Information, USA (NCBI) using BLAST search (http://blast.ncbi.nlm.nih.gov/Blast.cgi) to estimate the phylogenetic relationship. CLUSTAL X software (version 2.0, Conway Institute, USA) was used to generate alignment of endophytic fungi [40]. Phylogenetic analysis was carried out by the neighbor-joining method using MEGA software (version 4.0, Biodesign Institute, USA). The bootstrap was 1,000 replications to assess the reliable level to the nods of the tree [41].

\section{Primary screening of taxol-producing fungi based on PCR amplification}

The conserved sequences of three key genes in the taxol biosynthetic pathway, $t s, d b a t$, and bapt, were used as molecular markers to PCR amplification for primary screening of taxol-producing fungi. The specific primers ts-F, ts- $R$, dbat-F, dbat-R, bapt-F, bapt-R (Table 3) were synthesized by Sangon Biotech Co., Ltd. (Shanghai, China). PCR amplification was performed in a Mastercycler personal Thermal Cycler (Eppendorf Inc., Germany).The fungal isolates were firstly screened for the presence of ts gene, secondly screened for bapt gene, and lastly screened for dbat gene. PCR amplification was carried out according to previously reported PCR conditions in the literatures [16,17]. PCR products were analyzed on $2 \%$ (wt/vol) agarose gel and purified by DNA gel exaction kit (Axygen). The purified PCR products were ligated to pMD19-T vectors (TaKaRa), transformed into E. coli $\mathrm{DH} 10 \mathrm{~B}$, and sequenced by BGI-Shanghai. Those fungi with PCR positive for molecular makers were selected for the next screening.

\section{Determination of Taxol-producing fungi}

Three fungi with positive results of primary screening were inoculated into $250 \mathrm{ml}$ Erlenmeyer flasks containing
$25 \mathrm{ml}$ PDB medium to detect taxol production. The culture condition of fungal endophytes was the same as mentioned above, except that the culture time was changed to 5 days. The mycelia were harvested by centrifugation and freezed by liquid nitrogen, then thoroughly crushed in a mortar. The fermentation broths and ground mycelia were extracted with ethyl acetate 3 times at room temperature. All extracts were combined and concentrated under reduced pressure, and redissolved with $0.5 \mathrm{ml}$ of $100 \%$ methanol (v/v).

The extracts of each fungal isolate were examined for the presence of taxol using HPLC-MS. A $\mathrm{C}_{18}$ column $(4.6 \times 50 \mathrm{~mm}, 1.8 \mu \mathrm{m}$ particle size, Zorbax XDB, Agilent) was used to identify taxol by HPLC [11]. The methanol solution of putative taxol $(5 \mu \mathrm{l})$ were injected and elution was done with methanol $/ \mathrm{H}_{2} \mathrm{O}$ binary solvent-delivery gradient elution (0-20 $\mathrm{min}, 5 \%-100 \%$ methanol; 20-25 min, $100 \%$ methanol; 25-35 min, 5\% methanol; volume fraction). The wavelength at $226 \mathrm{~nm}$ was used to detect compounds eluting from the column. Electrospray mass spectroscopy was done on fungal taxol samples using the electrospray technique with an Agilent $1100 \mathrm{LC} /$ MSD trap. The sample in $100 \%$ methanol was injected with a spray flow of $2 \mu \mathrm{l} / \mathrm{min}$ and a spray voltage of 2.2 $\mathrm{kV}$ by the loop injection method. The mass spectral fragment ions of taxol are shown in Table 2.

\section{Nucleotide sequence accession numbers}

The partial sequences of the ITS rDNA, $t s$, and bapt genes obtained from cultures and clones were deposited in GenBank (NCBI) under the accession numbers JQ801635-JQ801669 and KC337343-KC337345.

\section{Competing interest}

The authors declare that they have no competing interest.

\section{Authors' contributions}

ZQX collected plant samples and designed the experiments; YYY isolated and characterized of endophytic fungi. NZ performed fungal cultivation. ZQX and $\mathrm{YW}$ were involved in conception and interpretation of the results and drafting the manuscript. ZQX and YW were involved in critically revision the manuscript and approved the manuscript for publication. All authors read and approved the final manuscript. 


\section{Acknowledgements}

This work was supported by the National Basic Research Program of China (973 Program, grant no. 2012CB721104), the National Natural Science Foundation of China (grants no. 31170101 and 31100073), and the major Projects of Knowledge Innovation Program of Chinese Academy of Sciences (grant no. KSCX2-EW-J-12).

\section{Author details}

${ }^{1}$ Key Laboratory of Synthetic Biology, Institute of Plant Physiology and Ecology, Shanghai Institutes for Biological Sciences, Chinese Academy of Sciences, Shanghai 200032, China. ${ }^{2}$ State Key Laboratory of Bioreactor Engineering, East China University of Science and Technology, Shanghai 200237, China.

Received: 12 November 2012 Accepted: 31 January 2013 Published: 28 March 2013

\section{References}

1. Kusari S, Spiteller M: Are we ready for industrial production of bioactive plant secondary metabolites utilizing endophytes? Nat Prod Rep 2011 28:1203-1207

2. Kusari S, Lamshoft M, Zuhlke S, Spiteller M: An endophytic fungus from Hypericum perforatum that produces hypericin. J Nat Prod 2008, 71:159-162.

3. Zhu D, Wang J, Zeng Q, Zhang Z, Yan R: A novel endophytic Huperzine Aproducing fungus, Shiraia sp. SIf14, isolated from Huperzia serrata. J Appl Microbiol 2010, 109:1469-1478.

4. Stierle A, Strobel G, Stierle D: Taxol and taxane production by Taxomyces andreanae, an endophytic fungus of Pacific yew. Science 1993, 260:214-216.

5. Zhou X, Zhu H, Liu L, Lin J, Tang K: A review: recent advances and future prospects of taxol-producing endophytic fungi. Appl Microbiol Biotechnol 2010, 86:1707-1717.

6. Pezzuto J: Taxol production in plant cell culture comes of age. Nat Biotechnol 1996, 14:1083

7. Nicolaou KC, Yang Z, Liu JJ, Ueno H, Nantermet PG, Guy RK, Claiborne CF, Renaud J, Couladouros EA, Paulvannan K, Sorensen EJ: Total synthesis of taxol. Nature 1994, 367:630-634.

8. Patel RN: Tour de paclitaxel: biocatalysis for semisynthesis. Annu Rev Microbiol 1998, 52:361-395.

9. Yukimune $Y$, Tabata $H$, Higashi $Y$, Hara Y: Methyl jasmonate-induced overproduction of paclitaxel and baccatin III in Taxus cell suspension cultures. Nat Biotechnol 1996, 14:1129-1132.

10. Flores-Bustamante ZR, Rivera-Orduna FN, Martinez-Cardenas A, Flores-Cotera LB: Microbial paclitaxel: advances and perspectives. J Antibiot 2010, 63:460-467.

11. Mirjalili MH, Farzaneh M, Bonfill M, Rezadoost H, Ghassempour A: Isolation and characterization of Stemphylium sedicola SBU-16 as a new endophytic taxol-producing fungus from Taxus baccata grown in Iran. FEMS Microbiol Lett 2012, 328:122-129.

12. Zhao K, Ping WX, Zhang LN, Liu J, Lin Y, Jin T, Zhou DP: Screening and breeding of high taxol producing fungi by genome shuffling. Sci China Ser C: Life Sci 2008, 51:222-231.

13. Wang Y, Guo B, Miao Z, Tang K: Transformation of taxol-producing endophytic fungi by restriction enzyme-mediated integration (REMI). FEMS Microbiol Lett 2007, 273:253-259.

14. Li JY, Sidhu RS, Bollon A, Strobel GA: Stimulation of taxol production in liquid cultures of Pestalotiopsis microspora. Mycol Res 1998, 102:461-464.

15. Ajikumar PK, Xiao WH, Tyo KE, Wang Y, Simeon F, Leonard E, Mucha O, Phon TH, Pfeifer B, Stephanopoulos G: Isoprenoid pathway optimization for Taxol precursor overproduction in Escherichia coli. Science 2010, 330:70-74.

16. Zhou $X$, Wang Z, Jiang K, Wei $Y$, Lin J, Sun $X$, Tang K: Screening of taxolproducing endophytic fungi from Taxus chinensis var. mairei. Appl Biochem Microbiol 2007, 43:490-494

17. Zhang P, Zhou PP, Jiang C, Yu H, Yu LJ: Screening of Taxol-producing fungi based on PCR amplification from Taxus. Biotechnol Lett 2008, 30:2119-2123.

18. Liu K, Ding X, Deng B, Chen W: Isolation and characterization of endophytic taxol-producing fungi from Taxus chinensis. J Ind Microbiol Biotechnol 2009, 36:1171-1177.
19. Caruso M, Colombo A, Fedeli L, Pavesi A, Quaroni S, Saracchi M, Ventrella G: Isolation of endophytic fungi and actinomycetes taxane producers. Annals Microbiol 2000, 50:3-14.

20. Strobel G, Daisy B: Bioprospecting for microbial endophytes and their natural products. Microbiol Mol Biol R 2003, 67:491-502.

21. Rakotoniriana EF, Munaut F, Decock C, Randriamampionona D, Andriambololoniaina M, Rakotomalala T, Rakotonirina EJ, Rabemanantsoa C, Cheuk K, Ratsimamanga SU, Mahillon J, El-Jaziri M, Quetin-Leclercq J, Corbisier AM: Endophytic fungi from leaves of Centella asiatica: occurrence and potential interactions within leaves. Antonie Van Leeuwenhoek 2008, 93:27-36.

22. Rubini MR, Silva-Ribeiro RT, Pomella AW, Maki CS, Araujo WL, Dos Santos $\mathrm{DR}$, Azevedo JL: Diversity of endophytic fungal community of cacao (Theobroma cacao L.) and biological control of Crinipellis perniciosa, causal agent of Witches' Broom Disease. Int J Biol Sci 2005, 1:24-33.

23. Croteau R, Ketchum RE, Long RM, Kaspera R, Wildung MR: Taxol biosynthesis and molecular genetics. Phytochem Rev 2006, 5:75-97.

24. Gangadevi V, Muthumary J: Isolation of Colletotrichum gloeosporioides, a novel endophytic taxol-producing fungus from the leaves of a medicinal plant, Justicia gendarussa. Mycol Balc 2008, 5:1-4.

25. Zhang P, Zhou PP, Yu $\sqcup$ : An endophytic taxol-producing fungus from Taxus media, Cladosporium cladosporioides MD2. Curr Microbiol 2009, 59:227-232.

26. Zhang P, Zhou PP, Yu $\sqcup$ : An endophytic taxol-producing fungus from Taxus $x$ media, Aspergillus candidus MD3. FEMS Microbiol Lett 2009, 293:155-159.

27. Staniek A, Woerdenbag HJ, Kayser O: Taxomyces andreanae: a presumed paclitaxel producer demystified? Planta Med 2009, 75:1561-1566.

28. Fitzpatrick DA: Horizontal gene transfer in fungi. FEMS Microbiol Lett 2012, $329: 1-8$

29. Li JY, Strobel G, Sidhu R, Hess WM, Ford EJ: Endophytic taxol-producing fungi from bald cypress, Taxodium distichum. Microbiol 1996, 142:2223-2226.

30. Kumaran RS, Muthumary J, Hur BK: Taxol from Phyllosticta citricarpa, a leaf spot fungus of the angiosperm Citrus medica. J Biosci Bioeng 2008, 106(1):103-106.

31. Gangadevi V, Muthumary J: Taxol production by Pestalotiopsis terminaliae, an endophytic fungus of Terminalia arjuna (arjun tree). Biotechnol Appl Biochem 2009, 52:9-15.

32. Gangadevi $V$, Muthumary J: A novel endophytic Taxol-producing fungus Chaetomella raphigera isolated from a medicinal plant, Terminalia arjuna. Appl Biochem Biotechnol 2009, 158:675-684.

33. Kumaran RS, Hur BK: Screening of species of the endophytic fungus Phomopsis for the production of the anticancer drug taxol. Biotechnol Appl Biochem 2009, 54:21-30.

34. Kumaran RS, Muthumary J, Hur BK: Isolation and identification of an anticancer drug, taxol from Phyllosticta tabernaemontanae, a leaf spot fungus of an angiosperm, Wrightia tinctoria. J Microbiol 2009, 47:40-49.

35. Tudzynski B: Gibberellin biosynthesis in fungi: genes, enzymes, evolution, and impact on biotechnology. Appl Microbiol Biotechnol 2005, 66:597-611.

36. Hedden P, Phillips AL, Rojas MC, Carrera E, Tudzynski B: Gibberellin Biosynthesis in Plants and Fungi: A Case of Convergent Evolution? J Plant Growth Regul 2001, 20:319-331.

37. Strobel G, Yang X, Sears J, Kramer R, Sidhu RS, Hess WM: Taxol from Pestalotiopsis microspora, an endophytic fungus of Taxus wallachiana. Microbiology 1996, 142:435-440.

38. Kim WK, Mauthe W, Hausner G, Klassen GR: Isolation of high-molecular -weight DNA and double-stranded RNAs from Fungi. Can J Bot 1990, 68:1898-1902.

39. Cookson BT, Chen YC, Eisner JD, Kattar MM, Rassoulian-Barrett SL, LaFe K, Yarfitz SL, Limaye AP: Identification of medically important yeasts using PCR-based detection of DNA sequence polymorphisms in the internal transcribed spacer 2 region of the rRNA genes. J Clin Microbiol 2000, 38:2302-2310.

40. Larkin MA, Blackshields G, Brown NP, Chenna R, McGettigan PA, McWilliam $H$, Valentin F, Wallace IM, Wilm A, Lopez R, et al: Clustal W and Clustal X version 2.0. Bioinformatics 2007, 23:2947-2948.

41. Tamura K, Dudley J, Nei M, Kumar S: MEGA4: Molecular Evolutionary Genetics Analysis (MEGA) software version 4.0. Mol Biol Evol 2007, 24:1596-1599.

doi:10.1186/1471-2180-13-71

Cite this article as: Xiong et al:: Diversity of endophytic fungi and screening of fungal paclitaxel producer from Anglojap yew, Taxus $x$ media. BMC Microbiology 2013 13:71. 